\title{
Acute kidney injury and acute-on-chronic liver failure classifications in prognosis assessment of patients with acute decompensation of cirrhosis
}

\author{
Paolo Angeli, ${ }^{1,2}$ Ezequiel Rodríguez, ${ }^{3,4,5}$ Salvatore Piano, ${ }^{1,2}$ Xavier Ariza, ${ }^{3,4,5}$ \\ Filippo Morando, ${ }^{1,2}$ Elsa Solà, ${ }^{3,4,5,6}$ Antonietta Romano, ${ }^{1,2}$ Elisabet García, ${ }^{7}$ \\ Marco Pavesi, ${ }^{6,7}$ Alessandro Risso, ${ }^{8}$ Alexander Gerbes, ${ }^{9}$ Chris Willars, ${ }^{10}$ \\ Mauro Bernardi, ${ }^{11}$ Vicente Arroyo, ${ }^{3,4,6}$ Pere Ginès, ${ }^{3,4,5,6}$ for the CANONIC Study \\ Investigators of the EASL-CLIF Consortium
}

\begin{abstract}
- Additional material is published online only. To view please visit the journal online (http://dx.doi.org/10.1136/ gutjnl-2014-307526).

For numbered affiliations see end of article.
\end{abstract}

Correspondence to Dr Pere Ginès, Liver Unit, Hospital Clinic of Barcelona, Barcelona, 08036, Spain; pgines@clinic.ub.es

Received 25 April 2014 Revised 17 September 2014 Accepted 18 September 2014 Published Online First 13 October 2014
CrossMark

To cite: Angeli $P$ Rodríguez E, Piano $S$, et al. Gut 2015;64:1616-1622.

\section{ABSTRACT}

Objective Prognostic stratification of patients with cirrhosis is common clinical practice. This study compares the prognostic accuracy (28-day and 90-day transplantfree mortality) of the acute-on-chronic liver failure (ACLF) classification (no ACLF, ACLF grades 1, 2 and 3) with that of acute kidney injury (AKI) classification (no AKI, AKI stages 1, 2 and 3).

Design The study was performed in 510 patients with an acute decompensation of cirrhosis previously included in the European Association for the Study of the LiverChronic Liver Failure consortium CANONIC study. ACLF was evaluated at enrolment and $48 \mathrm{~h}$ after enrolment, and AKI was evaluated at $48 \mathrm{~h}$ according to Acute Kidney Injury Network criteria.

Results 240 patients (47.1\%) met the criteria of ACLF at enrolment, while 98 patients (19.2\%) developed AKI. The presence of ACLF and AKI was strongly associated with mortality. 28-day transplant-free mortality and 90day transplant-free mortality of patients with ACLF $32 \%$ and $49.8 \%$, respectively) were significantly higher with respect to those of patients without $\operatorname{ACLF}(6.2 \%$ and $16.4 \%$, respectively; both $p<0.001)$. Corresponding values in patients with and without AKI were $46 \%$ and $59 \%$, and $12 \%$ and $25.6 \%$, respectively ( $p<0.0001$ for both). ACLF classification was more accurate than AKI classification in predicting 90-day mortality (area under the receiving operating characteristic curve $=0.72$ vs 0.62 ; $\mathrm{p}<0.0001$ ) in the whole series of patients. Moreover, assessment of ACLF classification at $48 \mathrm{~h}$ had significantly better prognostic accuracy compared with that of both AKI classification and ACLF classification at enrolment. Conclusions ACLF stratification is more accurate than AKI stratification in the prediction of short-term mortality in patients with acute decompensation of cirrhosis.

\section{INTRODUCTION}

The assessment of patients at risk of poor outcome is a crucial point in clinical practice since it helps the physician to decide on the intensity of treatment and monitoring and the most appropriate setting for patients' management (intensive care unit or regular ward). For this reason, interest in assessing organ dysfunction and severity of illness in hospitalised patients with cirrhosis has increased

\section{Significance of this study}

What is already known on this subject?

- Acute kidney injury (AKI) is a frequent complication of cirrhosis and a powerful predictor of death in hospitalised patients with liver cirrhosis.

- Acute-on-chronic liver failure (ACLF) is a clinical syndrome characterised by an acute deterioration of liver function and the development of organ failure in patients with chronic liver disease.

- The Chronic Liver Failure-Sequential Organ Failure Assessment (CLIF-SOFA) score has been shown to be a powerful predictor of death in hospitalised patients with cirrhosis.

What are the new findings?

- The development of ACLF according to CLIFSOFA score was found to be more accurate than AKI classification in the prediction of short-term mortality in patients with cirrhosis who are hospitalised for an acute decompensation of the liver disease.

- The assessment of CLIF-SOFA score after $48 \mathrm{~h}$ further improved the prognostic accuracy of ACLF classification.

- ACLF classification is able to predict the prognosis of patients with cirrhosis in a stage-dependent fashion, stratifying the mortality risk.

How might it impact on clinical practice in the foreseeable future?

- The ACLF classification is a simple tool able to identify patients with cirrhosis with high risk of short-term mortality.

- The ACLF classification may be able to identify high-risk patients requiring an intensive care management.

in recent years. This interest has grown in parallel with the development of the concept of acute-on-chronic liver failure (ACLF) that, in its 
simplest terms, is characterised by the abrupt onset of organ failures in patients with cirrhosis hospitalised for an acute decompensation of liver disease. ${ }^{1}$ The CLIF Acute-on-chronic liver failure in cirrhosis (CANONIC) study demonstrated that the presence of two (ACLF grade 2) or three organ failures (ACLF grade 3), which were defined by a Sequential Organ Failure Assessment (SOFA) score specifically adapted to patients with cirrhosis (CLIF-SOFA score) is associated with a high short-term mortality rate. ${ }^{1}$

In single-organ failure (ACLF grade 1), an increased mortality rate was observed for patients with kidney failure defined by a serum creatinine $(\mathrm{sCr})>2 \mathrm{mg} / \mathrm{dL}$ or with a failure of an organ other than the kidney when it is associated with an $\mathrm{sCr}$ $>1.5 \mathrm{mg} / \mathrm{dL}$ or with grade 1 or 2 hepatic encephalopathy. ${ }^{1}$ For many years, a cut-off level of $1.5 \mathrm{mg} / \mathrm{dL}$ of $\mathrm{sCr}$ has been used for the diagnosis of impairment of kidney function in patients with cirrhosis even when an increase of $\mathrm{sCr}$ with respect to baseline was considered. ${ }^{2-5}$ Consequently, the results of the CANONIC study have reinforced the concept that an impairment of kidney function has a marked negative impact on prognosis of hospitalised patients with cirrhosis, giving it a new dimension as a predictor of mortality.

In 2007, the Acute Kidney Injury Network (AKIN) developed a consensus definition of AKI, a new term to define acute renal failure according to AKIN criteria. ${ }^{6}$ The main innovative aspects introduced by AKIN criteria are the following: (a) an absolute increase in $\mathrm{sCr}$ is considered without any final cut-off value and (b) a staging system of AKI based on changes of $\mathrm{sCr}$ within $48 \mathrm{~h}$. These criteria are being used extensively in critically ill patients since they have been shown to be accurate in predicting prognosis. ${ }^{7}$ However, the information about AKIN criteria for diagnosis and classification of AKI in patients with cirrhosis is still limited. Recently, four prospective studies have shown that the AKI classification based on AKIN criteria predicts short-term and mid-term mortality in a stage-dependent fashion in hospitalised patients with cirrhosis. ${ }^{8-11}$ The results indicate that the AKI classification is useful in the prognostic stratification of patients with cirrhosis. Nevertheless, the AKI classification has so far not been compared with a more complete assessment of organ failures in these patients. Thus, the current study was designed to compare prospectively the AKI classification and the classification of ACLF in the prognostic stratification of hospitalised patients with acute decompensation of cirrhosis.

\section{PATIENTS AND METHODS}

\section{Study population}

The current study was performed in a population of patients included in the CANONIC study. ${ }^{1}$ The CANONIC study was a multicentre study aimed at evaluating the frequency, characteristics and outcome of ACLF in patients with cirrhosis admitted for an acute decompensation of the disease in 29 liver units from 8 European countries. Patients included in the current investigation were patients belonging to groups 1 and 2 of the CANONIC study. The distribution of patients in the CANONIC study has been reported in detail elsewhere. ${ }^{1}$ Briefly, patients in group 1 were patients admitted to hospital for an acute decompensation of cirrhosis who had at least one organ failure at admission to hospital, while patients in group 2 were patients admitted for an acute decompensation of cirrhosis but without organ failure that were chronologically enrolled after each patient with organ failure. In patients from both groups, clinical and laboratory data were collected at enrolment, 2 days after enrolment and at different time intervals throughout the hospitalisation, specifically
3-7, 8-14, 15-21 and 22-28 days after enrolment. Patients from group 3 of the CANONIC study were not included in the current investigation because laboratory data were collected at the time of enrolment but not throughout hospitalisation. A total of 639 patients from groups 1 and 2 were evaluated for inclusion in the current study. Of these patients, 129 were excluded because of at least one of the following reasons: lack of sCr values at day 2 (68 cases), lack of one or more variables included in the ACLF definition at day 2 (51 patients) and death or liver transplantation one day after enrolment (12 patients). Therefore, the current study was carried out in 510 patients. The distribution of patients is shown in online supplementary figure S1.

\section{Main variables and definitions}

The study was aimed at evaluating the AKI classification as well as the ACLF classification ${ }^{1} 12$ in determining the outcome of patients hospitalised for an acute decompensation of cirrhosis.

AKI was defined using the new Kidney Disease Improving Global Outcomes AKIN criteria and patients were categorised into 4 groups: no AKI and AKI stages 1, 2 and 3 (see online supplementary table S1). ${ }^{12}$ According to this classification, a patient was considered to developing AKI when there was an increase in $\mathrm{sCr} \geq 0.3 \mathrm{mg} / \mathrm{dL}$ or $\geq 50 \%$ in two different measurements obtained $48 \mathrm{~h}$ apart. The baseline sCr used was that obtained at enrolment of patients in the study while the second measurement was collected 2 days after enrolment.

ACLF was defined according to the presence and severity of organ failures as described in the CANONIC study, and patients were classified into 4 different groups: (1) no ACLF, (2) ACLF grade 1, (3) ACLF grade 2 and (4) ACLF grade 3 (see online supplementary table S1). ${ }^{1}$ Outcome was analysed as 28 -day and 90-day mortality.

\section{Statistical analysis}

Data were collected using an electronic case report form. Quantitative variables are reported as mean and SD or median, minimum and maximum according to their nature. Categorical variables are reported as count and percentage in each category and total. Factors associated with the development of AKI, ACLF and mortality were identified in a bivariate analysis with Student $t$ test and one-way analysis of variance for quantitative variables (depending on the number of categories), or the nonparametric corresponding tests, if needed. The 28-day and 90-day mortality rates were estimated as transplant-free mortality. In addition, since transplantation can be considered a competing event of death, curves showing the cumulative probability of failure were estimated with the cumulative incidence function proposed by Fine and Gray ${ }^{13}$ due to the lack of validity of the Kaplan-Meier estimator in this context.

The accuracy of AKI and ACLF classifications in predicting 28-day and 90-day transplantation-free mortality was assessed estimating the area under the curve of the receiving operating characteristic (AUCROC). Furthermore, they were compared in order to establish the better classification in prognosis. In a similar way, classification's accuracy for the estimation of both mortalities under a competing risk approach was assessed with the C-index and compared with the integrated discrimination improvement index. Two-sided $\mathrm{p}$ values of $<0.05$ were considered to indicate statistical significance.

\section{RESULTS}

\section{Baseline characteristics of the patients}

The demographic, clinical and laboratory characteristics of patients at the time of enrolment in the study are shown in table 1. 
Table 1 Baseline characteristics of patients at enrolment $(n=510)$

\begin{tabular}{|c|c|c|}
\hline Age (years) & $55 \pm 12$ & $(22-95)$ \\
\hline Male sex & $331(65)$ & \\
\hline \multicolumn{3}{|l|}{ Aetiology of cirrhosis } \\
\hline Alcohol & $292(60)$ & \\
\hline Hepatitis C virus & $67(13)$ & \\
\hline Alcohol plus hepatitis $C$ virus & $46(9)$ & \\
\hline Other causes & $84(18)$ & \\
\hline \multicolumn{3}{|l|}{ Previous decompensation(s) } \\
\hline Ascites & $310(60)$ & \\
\hline Encephalopathy & $152(32)$ & \\
\hline GI bleeding & $114(24)$ & \\
\hline Spontaneous bacterial peritonitis & $50(11)$ & \\
\hline \multicolumn{3}{|l|}{ Concomitant diseases } \\
\hline Arterial hypertension & $104(21)$ & \\
\hline Diabetes mellitus & $108(22)$ & \\
\hline Chronic renal failure & $45(9)$ & \\
\hline \multicolumn{3}{|l|}{ Cause(s) of admission } \\
\hline Ascites & $351(70)$ & \\
\hline Encephalopathy & $190(38)$ & \\
\hline Bacterial infection & $124(25)$ & \\
\hline GI bleeding & $79(16)$ & \\
\hline Other & $177(35)$ & \\
\hline \multicolumn{3}{|l|}{ Exploratory data } \\
\hline Mean arterial pressure $(\mathrm{mm} \mathrm{Hg})$ & $82 \pm 12$ & $(46-122)$ \\
\hline Heart rate (bpm) & $83 \pm 17$ & $(45-150)$ \\
\hline \multicolumn{3}{|l|}{ Laboratory data } \\
\hline White blood cells $\left(\times 10^{9} / \mathrm{L}\right)$ & $9 \pm 5$ & $(0.8-50)$ \\
\hline Platelet count $\left(\times 10^{9} / \mathrm{L}\right)$ & $102 \pm 7$ & $(12-543)$ \\
\hline Serum bilirubin (mg/dL) & $8 \pm 9$ & $(0.5-45.5)$ \\
\hline Serum albumin (g/dL) & $2.8 \pm 0.5$ & $(1.3-4.5)$ \\
\hline International normalised ratio & $1.9 \pm 0.7$ & $(1-7)$ \\
\hline Plasma C-reactive protein (mg/L) & $38 \pm 42$ & $(0.6-326)$ \\
\hline Serum creatinine $(\mathrm{mg} / \mathrm{dL})$ & $1.6 \pm 1.3$ & $(0.4-12.5)$ \\
\hline Serum sodium (mmol/L) & $134 \pm 6$ & $(111-153)$ \\
\hline Child-Pugh score & $10 \pm 2$ & $(5-15)$ \\
\hline MELD & $22 \pm 8$ & $(6-40)$ \\
\hline
\end{tabular}

Data are expressed as mean \pm SD (min-max) or number of patients (percentage).

MELD, model for end-stage liver disease.

The majority of patients had advanced cirrhosis as indicated by markedly impaired liver function tests and high Child-Pugh and model for end-stage liver disease (MELD) scores.

\section{Frequency and characteristics of AKI and relationship with outcome}

In total, 98 of the 510 patients (19.2\%) developed AKI during the first $48 \mathrm{~h}$ after enrolment. In these patients, $\mathrm{sCr}$ concentration increased from $2.5 \pm 1.9$ at baseline to $2.7 \pm 1.9 \mathrm{mg} / \mathrm{dL}$ at day $2(p=0.0064)$. Corresponding values of $\mathrm{sCr}$ in the 412 patients who did not develop AKI were $1.4 \pm 1.1$ and 1.2 $\pm 0.9 \mathrm{mg} / \mathrm{dL}$, respectively $(\mathrm{p}<0.0001)$. In total, 34 of 98 patients (34.7\%) who developed AKI had AKI stage 1, 4 (4.1\%) stage 2 and $60(61.2 \%)$ stage 3 . The relatively high proportion of patients with AKI stage 3 was largely due to the need for renal replacement therapy in 50 of 60 patients $(83.3 \%)$ who had AKI stage 3. Values of sCr according to the different AKI stages are shown in online supplementary figure S2.

Table 2 shows the comparison of baseline characteristics of patients categorised according to the subsequent development of AKI within the $48 \mathrm{~h}$ time frame. Patients who developed AKI
Table 2 Baseline characteristics of patients at enrolment according to the subsequent development of acute kidney injury (AKI) at $48 \mathrm{~h}$ after enrolment

\begin{tabular}{|c|c|c|c|}
\hline & No AKI $(n=412)$ & AKI $(n=98)$ & p Value \\
\hline Age (years) & $56 \pm 11$ & $53 \pm 14$ & 0.09 \\
\hline Male sex & $270(65)$ & $61(62)$ & 0.53 \\
\hline \multicolumn{4}{|l|}{ Aetiology of cirrhosis } \\
\hline Alcohol & $243(61)$ & $49(52)$ & 0.07 \\
\hline Hepatitis C virus & $56(14)$ & $11(12)$ & 0.50 \\
\hline Alcohol plus hepatitis C virus & $37(9)$ & $9(9)$ & 0.98 \\
\hline Other causes & $58(15)$ & $26(27)$ & 0.003 \\
\hline \multicolumn{4}{|l|}{ Previous decompensation(s) } \\
\hline Ascites & $250(65)$ & $60(63)$ & 0.79 \\
\hline Encephalopathy & $126(33)$ & $26(28)$ & 0.36 \\
\hline GI bleeding & $98(26)$ & $16(17)$ & 0.09 \\
\hline Spontaneous bacterial peritonitis & $41(11)$ & $9(10)$ & 0.74 \\
\hline \multicolumn{4}{|l|}{ Concomitant diseases } \\
\hline Arterial hypertension & $76(19)$ & $28(29)$ & 0.03 \\
\hline Diabetes mellitus & $83(20)$ & $25(25)$ & 0.28 \\
\hline Chronic renal failure & $36(9)$ & $9(9)$ & 0.93 \\
\hline \multicolumn{4}{|l|}{ Cause(s) of admission } \\
\hline Ascites & $274(68)$ & $77(79)$ & 0.02 \\
\hline Encephalopathy & $142(35)$ & $48(49)$ & 0.01 \\
\hline Bacterial infection & $95(24)$ & $29(30)$ & 0.23 \\
\hline GI bleeding & $68(17)$ & $11(11)$ & 0.18 \\
\hline Other & $147(36)$ & $30(31)$ & 0.27 \\
\hline \multicolumn{4}{|l|}{ Exploratory data } \\
\hline Mean arterial pressure $(\mathrm{mm} \mathrm{Hg})$ & $82 \pm 12$ & $79 \pm 12$ & 0.007 \\
\hline Heart rate (beats/min) & $83 \pm 17$ & $87 \pm 19$ & 0.02 \\
\hline \multicolumn{4}{|l|}{ Laboratory data } \\
\hline White blood cells $\left(\times 10^{9} / \mathrm{L}\right)$ & $8.2 \pm 5.3$ & $10.4 \pm 7.2$ & 0.0005 \\
\hline Platelet count $\left(\times 10^{9} / \mathrm{L}\right)$ & $103 \pm 72$ & $99 \pm 65$ & 0.84 \\
\hline Serum bilirubin (mg/dL) & $7 \pm 8.2$ & $12 \pm 12$ & 0.0004 \\
\hline Serum albumin ( $g / d L)$ & $2.8 \pm 0.6$ & $2.7 \pm 0.6$ & 0.26 \\
\hline International normalised ratio & $1.8 \pm 1$ & $2.2 \pm 1$ & $<0.0001$ \\
\hline Plasma C-reactive protein (mg/L) & $35 \pm 39$ & $52 \pm 50$ & 0.0003 \\
\hline Serum creatinine (mg/dL) & $1.4 \pm 1.1$ & $2.5 \pm 2$ & $<0.0001$ \\
\hline Serum sodium (mmol/L) & $135 \pm 6$ & $133 \pm 6$ & 0.06 \\
\hline Child-Pugh score & $10 \pm 2$ & $11 \pm 2$ & $<0.0001$ \\
\hline MELD & $21 \pm 7$ & $28 \pm 87$ & $<0.0001$ \\
\hline
\end{tabular}

Data are expressed as mean \pm SD or number of patients (percentage).

MELD, model for end-stage liver disease.

had more frequent history of arterial hypertension, higher frequency of ascites and hepatic encephalopathy, and lower mean arterial pressure and higher heart rate compared with patients who did not develop AKI. Moreover, patients who developed AKI had more severe impairment of liver function, higher $\mathrm{sCr}$ levels and higher leucocyte count and C-reactive protein levels compared with those of patients who did not develop AKI. The frequency of ascites and encephalopathy and the magnitude of changes in laboratory tests paralleled the presence and severity of AKI (see online supplementary table S2).

The development of AKI was associated with precipitating factors in most patients, particularly bacterial infections and volume depletion. In addition, 15 of 98 patients had type 1 hepatorenal syndrome, according to the International Ascites $\mathrm{Club}^{14}{ }^{15}$ (table 3).

The presence of AKI at $48 \mathrm{~h}$ after enrolment had a strong association with prognosis in a stage-dependent manner. Twenty-eight-day transplant-free mortality in patients with AKI 
Table 3 Potential causes of acute kidney injury

\begin{tabular}{lc}
\hline Volume depletion & 21 (17) \\
Gl bleeding & 16 \\
Diarrhoea & 3 \\
Diuretic overdose & 2 \\
Bacterial infection & 47 (39) \\
Spontaneous bacterial peritonitis & 17 \\
Pneumonia & 9 \\
Urinary tract infection & 7 \\
Skin infection & 4 \\
Other infections & 4 \\
Unproved suspected infection & 6 \\
Type 1 hepatorenal syndrome & $15(12)$ \\
Surgery & 2 (2) \\
Unknown & $37(30)$ \\
\hline Values are number of patients and percentages (in parentheses). \\
Patients could have more than one potential cause: 41 patients had one, 19 had two \\
and 1 had three. The cause was unknown in 37 patients.
\end{tabular}

stage 2-3 was 55.9\% compared with $25 \%$ in patients with AKI stage 1 and $12 \%$ in patients without AKI $(p<0.0001)$. Corresponding values of 90-day transplant-free mortality were $67.3 \%, 40 \%$ and $25.6 \%$, respectively $(\mathrm{p}<0.0001)$. During the 90-day period after enrolment, 63 patients $(12.5 \%)$ were transplanted, 17 with AKI and 46 without AKI (17.5\% and 11.4\% from the AKI and non-AKI groups, respectively; $p=0.09$ ).

Because transplantation can be considered a competing event of death, the cumulative incidence function of mortality during follow-up was assessed using a competing risk approach (see the Methods section). As shown in figure 1, presence and severity of AKI was clearly associated with 28-day and 90-day cumulative mortality risk.

\section{ACLF at enrolment and relationship with outcome}

In total, 240 of 510 patients (47.1\%) met the criteria of ACLF at enrolment in the study: 110 had ACLF grade 1 (45.8\%), 96 grade $2(40 \%)$ and $34(14.2 \%)$ grade 3 . The comparison of the baseline characteristics of patients with and without ACLF is shown in table 4. Patients with ACLF at enrolment had higher frequency of ascites and hepatic encephalopathy at admission and lower frequency of GI bleeding compared with patients without ACLF. Surprisingly, there were no significant differences between the two groups with respect to the frequency of bacterial infections. Patients with ACLF had more frequent history of arterial hypertension and chronic kidney disease, and more severe impairment of liver function tests, higher $\mathrm{sCr}$, lower
Table 4 Baseline characteristics of patients according to the presence of acute-on-chronic liver failure (ACLF) at enrolment

\begin{tabular}{|c|c|c|c|}
\hline & $\begin{array}{l}\text { No ACLF } \\
(n=270)\end{array}$ & $\begin{array}{l}\text { ACLF } \\
(n=240)\end{array}$ & $p$ Value \\
\hline Age (years) & $55 \pm 12$ & $55 \pm 11$ & 0.46 \\
\hline Male sex & $173(64)$ & $158(34)$ & 0.68 \\
\hline \multicolumn{4}{|l|}{ Aetiology of cirrhosis } \\
\hline Alcohol & $151(58)$ & $141(61)$ & 0.49 \\
\hline Hepatitis C virus & $39(15)$ & $28(12)$ & 0.35 \\
\hline Alcohol plus hepatitis C virus & $24(9)$ & $22(0.4)$ & 0.91 \\
\hline Other causes & $45(17)$ & $39(17)$ & 0.90 \\
\hline \multicolumn{4}{|l|}{ Previous decompensation(s) } \\
\hline Ascites & $161(63)$ & $149(66)$ & 0.49 \\
\hline Encephalopathy & $79(31)$ & $73(33)$ & 0.62 \\
\hline GI bleeding & $62(24)$ & $52(24)$ & 0.81 \\
\hline Spontaneous bacterial peritonitis & $26(10)$ & $24(11)$ & 0.83 \\
\hline \multicolumn{4}{|l|}{ Concomitant diseases } \\
\hline Arterial hypertension & $46(17)$ & $58(24)$ & 0.04 \\
\hline Diabetes mellitus & $54(20)$ & $54(23)$ & 0.48 \\
\hline Chronic renal failure & $14(5)$ & $31(13)$ & 0.002 \\
\hline \multicolumn{4}{|l|}{ Cause(s) of admission } \\
\hline Ascites & $170(64)$ & $181(76)$ & 0.003 \\
\hline Encephalopathy & $81(30)$ & $109(46)$ & 0.0003 \\
\hline Bacterial infection & $59(23)$ & $65(29)$ & 0.13 \\
\hline GI bleeding & $51(19)$ & $28(12)$ & 0.02 \\
\hline Other & $94(35)$ & $83(35)$ & 0.99 \\
\hline \multicolumn{4}{|l|}{ Exploratory data } \\
\hline Mean arterial pressure $(\mathrm{mm} \mathrm{Hg})$ & $84 \pm 11$ & $79 \pm 13$ & $<0.0001$ \\
\hline Heart rate (beats/min) & $84 \pm 16$ & $83 \pm 18$ & 0.63 \\
\hline \multicolumn{4}{|l|}{ Laboratory data } \\
\hline White blood cells $\left(\times 10^{9} / \mathrm{L}\right)$ & $7.3 \pm 4.4$ & $10.1 \pm 6.7$ & $<0.0001$ \\
\hline Platelet count $\left(\times 10^{9} / \mathrm{L}\right)$ & $104 \pm 70$ & $101 \pm 73$ & 0.35 \\
\hline Serum bilirubin (mg/dL) & $5.6 \pm 6.3$ & $11 \pm 11$ & $<0.0001$ \\
\hline Serum albumin (g/dL) & $2.7 \pm 0.6$ & $2.8 \pm 0.6$ & 0.14 \\
\hline International normalised ratio & $1.7 \pm 0.6$ & $2.1 \pm 0.9$ & $<0.0001$ \\
\hline Plasma C-reactive protein (mg/L) & $33 \pm 39$ & $45 \pm 0.6$ & 0.0009 \\
\hline Serum creatinine (mg/dL) & $1.0 \pm 0.4$ & $2.4 \pm 1.6$ & $<0.0001$ \\
\hline Serum sodium (mmol/L) & $135 \pm 6$ & $134 \pm 6$ & 0.004 \\
\hline Child-Pugh score & $10 \pm 2$ & $11 \pm 2$ & $<0.0001$ \\
\hline MELD & $18 \pm 6$ & $27 \pm 7$ & $<0.0001$ \\
\hline
\end{tabular}

Data are expressed as mean \pm SD or number of patients (percentage).

MELD, model for end-stage liver disease.

serum sodium and arterial pressure, and higher white blood cell count and plasma C-reactive protein levels at enrolment with respect to patients without ACLF (table 4). The frequency of
Figure 1 Probability of survival of all patients included, categorised according to the presence and severity of acute kidney injury (AKI) $48 \mathrm{~h}$ after enrolment. Calculations were performed using competing risks approach (see text). (A) 28-day survival; (B) 90-day survival.
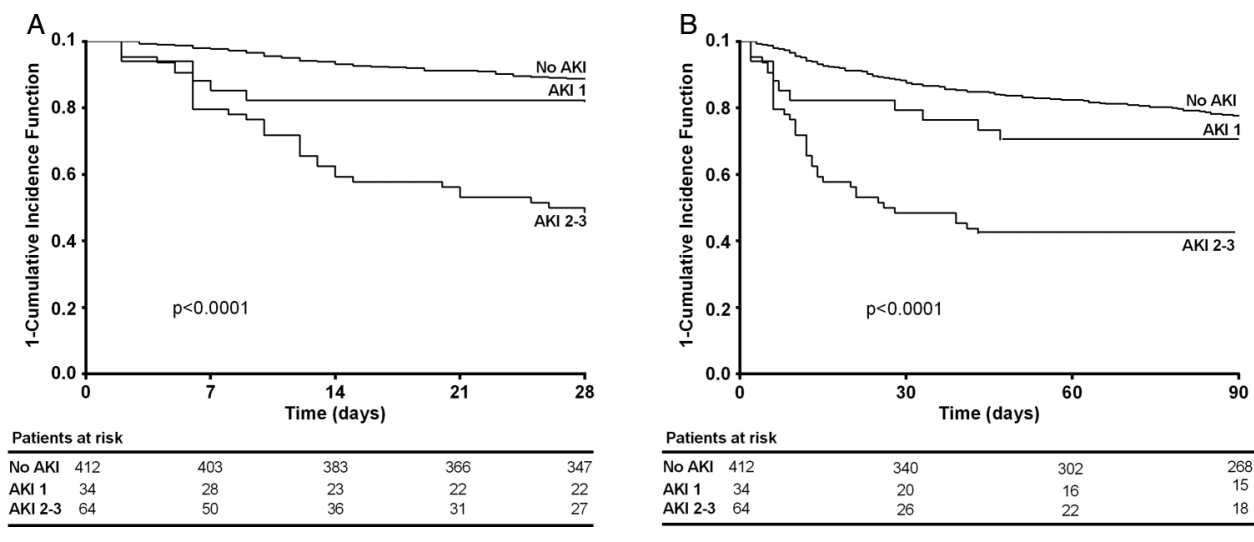
Figure 2 Probability of survival of all patients included, categorised according to the presence and severity of acute-on-chronic-liver-failure (ACLF) at enrolment. Calculations were performed using competing risks approach (see text). (A) 28-day survival; (B) 90-day survival.

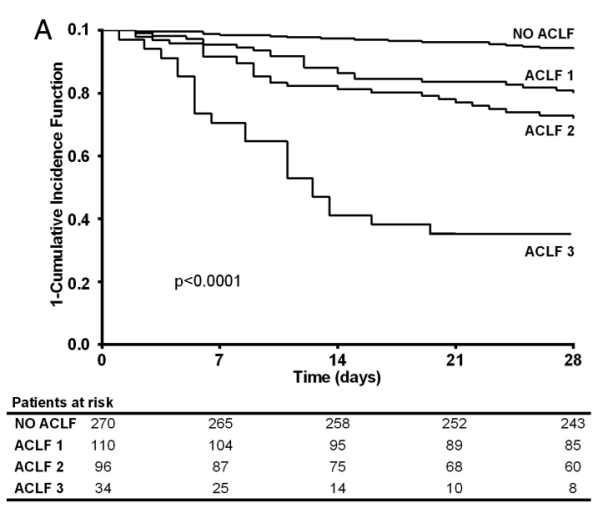

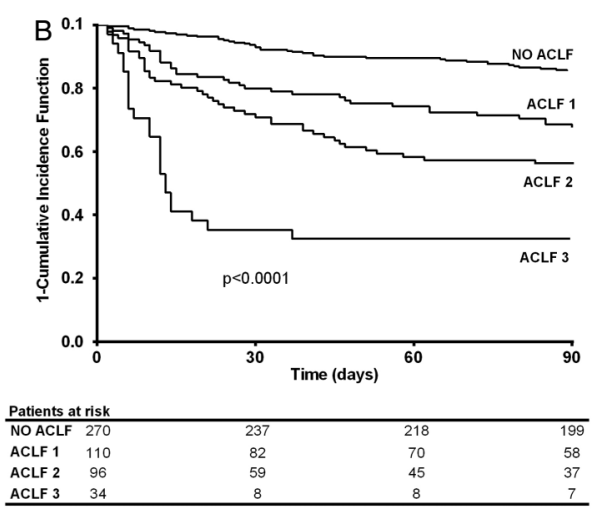

ascites and encephalopathy and the magnitude of changes in laboratory tests changed in parallel with severity of ACLF (see online supplementary table S3).

The frequency and stages of ACLF in patients categorised according to the absence or presence of AKI and its stage are shown in online supplementary figure S3.

The presence of ACLF at enrolment was strongly associated with mortality. Twenty-eight-day transplant-free mortality in patients with ACLF was $32 \%$ compared with only $6.2 \%$ in patients without ACLF $(\mathrm{p}<0.0001)$. Corresponding values of 90-day transplant-free mortality were $49.8 \%$ and $16.4 \%$, respectively $(\mathrm{p}<0.001)$. During the 3 -month period after enrolment, 35 (14.6\%) patients with ACLF and 28 (10.4\%) patients without ACLF were transplanted $(p=0.15)$. In a way similar to the analysis performed with AKI, the cumulative incidence function of mortality during follow-up of all patients categorised according to the presence and severity of ACLF was assessed using risk estimates adjusted to the competing risk of transplantation. As shown in figure 2, presence and severity of ACLF was clearly associated with 28-day and 90-day cumulative mortality risk.

\section{Comparison of the prognostic value of AKI classification vs ACLF classification}

We then compared the accuracy of the two classifications in predicting 28-day and 90-day mortality in the whole series of patients admitted to hospital for management of an acute decompensation of cirrhosis. The AUCROC for predicting transplant-free mortality was significantly better for the ACLF classification compared with that of the AKI classification, both at 28 and 90 days (table 5). Likewise, the comparison of the two classifications under a competing risk analysis approach was also better for ACLF compared with AKI.
We then analysed whether assessment of presence and severity of ACLF after 2 days of enrolment (that is, at the same day that the AKI classification was assessed) had better prognostic accuracy than assessment of ACLF at enrolment. During the 2-day period after enrolment, 28 of 270 patients (10.4\%) who did not have ACLF at baseline developed ACLF (grade 1 in 16 patients, grade 2 in 11 patients and grade 3 in 1 patient). By contrast, 64 of 240 patients $(26.7 \%)$ who had ACLF at enrolment improved and no longer met the criteria of ACLF at day 2 (previous ACLF grades were 1 in 43 patients, 2 in 18 patients and 3 in 3 patients). Assessment of ACLF classification at day 2 had significantly better prognostic accuracy compared with those of AKI classification and ACLF classification at enrolment, both using comparisons of standard AUCROC curves for transplant-free mortality as well as comparisons under a competing risk approach considering transplant as a competing event of death (table 5). The predictive accuracy of the model was similar according to the different aetiologies of cirrhosis (alcoholic cirrhosis vs hepatitis $\mathrm{C}$ or $\mathrm{B}$ infection).

\section{Causes of death and effect of bacterial infections}

Table 6 shows the causes of death during the 28-day period in patients classified according to the presence or absence of ACLF at $48 \mathrm{~h}$ after enrolment. As expected, mortality was markedly higher in patients with ACLF than in those without $(\mathrm{p}<0.0001)$. There were no significant differences between the two groups with respect to causes of death.

Among the 510 patients included, 150 developed a bacterial infection (29.4\%). The prevalence of infection increased with the presence and severity of ACLF. More importantly, mortality in patients with infections correlated also with presence and severity of ACLF (table 7).

Table 5 Comparison of acute kidney injury (AKI) and acute-on-chronic liver failure (ACLF) classifications to predict 28-day and 90-day mortality

\begin{tabular}{|c|c|c|c|c|c|c|}
\hline & AKI & ACLF at enrolment & ACLF at $48 \mathrm{~h}$ & $\begin{array}{l}\text { AKI vs ACLF } \\
\text { at enrolment }\end{array}$ & AKI vs ACLF at $48 \mathrm{~h}$ & $\begin{array}{l}\text { ACLF at enrolment } \\
\text { vs ACLF at } 48 \mathrm{~h}\end{array}$ \\
\hline AUCROC* & & & & & $p$ Value & \\
\hline 28-day & 0.68 (0.62 to 0.73 ) & 0.77 (0.71 to 0.82 ) & 0.84 (0.80 to 0.89 ) & 0.0049 & $<0.0001$ & 0.0021 \\
\hline 90-day & 0.62 (0.57 to 0.66$)$ & 0.72 (0.67 to 0.77$)$ & 0.77 (0.73 to 0.82 ) & $<0.0001$ & $<0.0001$ & 0.0092 \\
\hline C-indext & & & & & $p$ Value & \\
\hline 28-day & 0.66 (0.61 to 0.71 ) & 0.74 (0.69 to 0.79 ) & 0.81 (0.76 to 0.85 ) & 0.09 & $<0.0001$ & 0.0004 \\
\hline 90-day & 0.61 (0.57 to 0.65 ) & 0.69 (0.65 to 0.73$)$ & 0.74 (0.70 to 0.77 ) & 0.0028 & $<0.0001$ & 0.0002 \\
\hline
\end{tabular}

Values in parentheses are $95 \%$ Cls.

*Transplant-free mortality.

tMortality considering transplantation as competing event.

AUCROC, area under the curve of the receiving operating characteristic. 
Table 6 Causes of death in the whole series categorised according to the presence or absence of acute-on-chronic liver failure (ACLF) at $48 \mathrm{~h}$ after enrolment

\begin{tabular}{lcc}
\hline Cause & No ACLF $(\mathbf{n}=\mathbf{3 0 6})$ & ACLF $(\mathbf{n}=\mathbf{2 0 4})$ \\
\hline Septic shock & $3(25)$ & $32(42)$ \\
Multiorgan failure & $3(25)$ & $22(29)$ \\
Hypovolemic shock & $2(17)$ & $12(16)$ \\
Other & $0(0)$ & $7(9)$ \\
Unknown & $4(33)$ & $3(4)$ \\
Total & $12(4)$ & $76(37)$ \\
\hline
\end{tabular}

Data are expressed as number of patients (values in parentheses are percentages). Percentages in 'Total' refer to the number of patients in each group (no ACLF and ACLF). In the other rows percentages refer to the number of deaths in each group (no ACLF vc ACLF).

\section{DISCUSSION}

The purpose of the current study was to compare the accuracy of the AKI classification with that of the ACLF classification, recently proposed by the European Association for the Study of the Liver (EASL)-CLIF consortium on the basis of the CANONIC study, in the prediction of 28-day and 90-day mortality in patients hospitalised for an acute decompensation of cirrhosis. ${ }^{1}$

The main result of the study is that the ACLF classification has greater prognostic accuracy compared with that of the AKI classification in the prediction of 28-day and 90-day mortality. This holds true considering the ACLF classification at enrolment, but more especially when patients were stratified according to the ACLF classification at $48 \mathrm{~h}$ of enrolment. This greater prognostic accuracy of the ACLF system is first of all due to the fact that, in contrast to the AKIN criteria, it includes in its definition also non-kidney organ failures such as encephalopathy and cardiovascular dysfunction that are known to have a strong negative impact on prognosis of patients with cirrhosis. In addition, the diagnosis of ACLF also includes liver and coagulation failures, based on the use of serum bilirubin and international normalised ratio, respectively, that are known to have a strong predictive value on 90-day mortality in these patients. ${ }^{16-18}$ The availability of a classification system, like the current one on ACLF, which is simple in its application and also able to provide an accurate prognostic assessment at the point of enrolment, is a key factor for planning the management of a severe clinical condition like acute decompensation of cirrhosis. In particular, the identification of patients with a high risk of mortality would be invaluable in deciding when and in which patients to intensify medical care, and in selecting those for liver transplantation. Recognition of high-risk patients with an acute decompensation of the liver disease could facilitate focused resource allocation by identifying

Table 7 Prevalence and mortality of bacterial infections in patients categorised according to acute-on-chronic liver failure (ACLF) grades

\begin{tabular}{lccccc}
\hline & $\begin{array}{l}\text { No ACLF } \\
(\mathbf{n = 3 0 6 )}\end{array}$ & $\begin{array}{l}\text { ACLF } \\
\text { grade 1 } \\
(\mathbf{n}=92)\end{array}$ & $\begin{array}{l}\text { ACLF } \\
\text { grade 2 } \\
(\mathbf{n}=66)\end{array}$ & $\begin{array}{l}\text { ACLF } \\
\text { grade 3 } \\
(\mathbf{n}=46)\end{array}$ & p Value \\
\hline $\begin{array}{l}\text { Bacterial infection } \\
\text { Death }\end{array}$ & $82(27)$ & $30(33)$ & $16(25)$ & $22(49)$ & 0.017 \\
28-day & $3(4)$ & $7(23)$ & $7(44)$ & $15(68)$ & $<0.0001$ \\
90-day & $14(17)$ & $10(35)$ & $9(56)$ & $17(77)$ & $<0.0001$ \\
\hline \multicolumn{5}{l}{ Data are expressed as number of patients (values in parentheses are percentages). }
\end{tabular}

Angeli P, et al. Gut 2015;64:1616-1622. doi:10.1136/gutjnl-2014-307526 those most likely to benefit. Finally, subsequent research in this field can help stratify diagnostic and therapeutic findings between high-risk and low-risk subsets of patients, thereby promoting a targeted evaluation and/or application of them.

Approximately $20 \%$ of the population of the current study developed AKI within the first $48 \mathrm{~h}$ after enrolment. The design of the CANONIC study may have favoured an underestimation of the actual prevalence of AKI in our series of patients. In fact, according to the definition of the AKIN criteria, only changes in $\mathrm{sCr}$ within the first $48 \mathrm{~h}$ after enrolment were considered for the definition of AKI. Therefore, patients who could theoretically have developed AKI before or after this interval of time were not considered. Looking at the baseline $\mathrm{sCr}$ in patients without AKI (see online supplementary figure S2) it is possible that some patients could have developed AKI before enrolment and probably before admission to hospital. It has been reported that a community-acquired AKI may account for at least one-third of all AKI episodes detected in general population. ${ }^{19}$ However, the detection of community-acquired AKI criteria requires an $\mathrm{sCr}$ value to be evaluated just prior to hospital admission or a surrogate for it. Thus, the lack of this value represents more a limitation of applicability of the AKIN criteria than of the study design. As far as the possibility of development of AKI later during the hospitalisation, it should be taken into account that it has been observed that most AKI episodes occur during the first $48 \mathrm{~h}$ after admission. ${ }^{20}$ On the other hand, the possibility that some patients could have developed ACLF more than $48 \mathrm{~h}$ after enrolment should also be taken into account. Thus, the comparison limited to the first $48 \mathrm{~h}$, that is, at the same day that the AKI classification was assessed, puts the two classification systems in a condition of virtual equality. Despite this equality, the ACLF classification made it possible to stratify in prognostic terms $47.1 \%$ of patients at enrolment and $40 \%$ of patients after $48 \mathrm{~h}$. A number of factors could have contributed to this high frequency of ACLF. First, among patients who had an organ failure at enrolment those with an organ failure other than the kidney were $41.7 \%$. Second, the use of an absolute value of $\mathrm{sCr} \geq 1.5 \mathrm{mg} / \mathrm{dL}$, which has always shown a high prognostic value in hospitalised patients with cirrhosis, without suffering the methodological limitations of the AKIN criteria, has contributed to define an additional $10.4 \%$ of patients with ACLF. Finally, to fully understand the significance of this marked difference in frequency between AKI and ACLF in our patients, it is necessary to add that among the patients in whom AKI was diagnosed only $15.3 \%$ did not have ACLF (see online supplementary figure S3).

One downside of the study appeared when trying to analyse the prognostic accuracy of the model based on ACLF classification for prediction of adverse events. Unfortunately, this analysis could not be done because the number of patients without adverse events in the current series of patients was very low (only $10 \%$ of the series), which precluded a conclusive statistical analysis. Moreover, when adverse events related to organ failures were considered, there was a problem that organ failures were included in the definition of ACLF, which made the analysis not possible.

A final aspect of the study that should be emphasised is that the prognostic stratification of patients was done in the first $48 \mathrm{~h}$ after admission. However, this is an important period of time in which clinical decisions must be taken.

In conclusion, the ACLF classification provides a simple tool for an immediate stratification of patients with acute decompensation of cirrhosis on hospital admission. The prognostic accuracy of ACLF classification can be further enhanced when it is 
applied $48 \mathrm{~h}$ after admission. The ACLF classification both at admission and at $48 \mathrm{~h}$ has better prognostic accuracy than the AKI classification, thus it should be used in the prognostic stratification of these patients.

\section{Author affiliations}

${ }^{1}$ Department of Medicine (DIMED), University of Padova, Italy

${ }^{2}$ Unit of Hepatic Emergencies and Liver Transplantation, Padova, Italy

${ }^{3}$ Liver Unit, Hospital Clinic de Barcelona, University of Barcelona, Spain

${ }^{4}$ Institut d'Investigacions Biomediques Agust Pi i Sunyer (IDIBAPS), Barcelona, Spain

${ }^{5}$ Fundación Renal Iñigo Alvarez de Toledo, (FRIAT), Madrid, Spain

${ }^{6}$ Centro de Investigación Biomédica en Red en Enfermedades Hepáticas y Digestivas (CIBEREHD), Spain

${ }^{7}$ Data Management Centre, CLIF Consortium, Barcelona, Spain

${ }^{8}$ Hospital San Giovanni Battista Hospital, University of Torino, Italy

${ }^{9}$ Liver Unit, Klinikum Munich, Lugwig Maximilian University of Munich, Germany

${ }^{10}$ Intensive Care Unit, Hepatology Department, Kings College London, UK

${ }^{11}$ Semeiotica Medica-Policlinico S. Orsola-Malpighi, University of Bologna, Italy

Acknowledgements The work of physicians and nurses working in the different hospitals that participated in the CANONIC study is greatly appreciated. This is study number 3 of the CANONIC Project.

Contributors Design of study: PA and PG. Data collection: ER, SP, XA, FM, ES, AnR, AIR and CW. Analysis of data: PA, PG, EG, MP, ER and XA. Drafting and writing of manuscript: $P A, P G, E R, S P$ and $X A$. Critical revision of data and manuscript revision: $A G, C W, M B, V A, P A$ and $P G$.

Funding Ministerio de Economia y Competitividad, Fondo de Investigación Sanitaria Instituto de Salud Carlos III (FIS 2012/ PI12/00330).

Competing interests This study did not receive any direct funding; however, the CLIF consortium is supported by an unconditional grant from GRIFOLS S.A. Spain. PG has received research funding from the Instituto de Salud Carlos III (FIS 2012/ PI12/00330). PG has also worked on a consultancy basis for Sequana Medical AG and Ferring Pharmaceuticals. He has also received research grants from Sequana Medical AG and Grifols S.A not related to this paper. VA has received grant and research support from Grifols S.A. MB declares he has acted as speaker for the following companies entirely unrelated to this study: CLS Behring GmbH, PPTA Europe and Baxter Healthcare SA. He has worked on a consultancy basis for CLS Behring GmbH and Baxter Healthcare SA.

Patient consent Obtained.

Ethics approval Each hospital obtained approval.

Provenance and peer review Not commissioned; externally peer reviewed.

Data sharing statement This is an original research article. The data base from which the data were obtained for this study is available in the Data base Management Center of the EASL-CLIF consortium, University of Barcelona, Spain. The data base is available for all the investigators who are involved or who will be involved in research studies after the approval of the EASL-CLIF consortium Steering Committee.

\section{REFERENCES}

1 Moreau $R$, Jalan $R$, Ginès $P$, et al. Acute-on-chronic liver failure is a distinct syndrome that develops in patients with acute decompensation of cirrhosis. Gastroenterology 2013;144:1426-37.

2 Ginès $P$, Arroyo $V$, Vargas $V$, et al. Paracentesis with intravenous infusion of albumin as compared with peritoneovenous shunting in cirrhosis with refractory ascites. N Engl J Med 1991;325:829-35.

3 Sort $\mathrm{P}$, Navasa $\mathrm{M}$, Arroyo $\mathrm{V}$, et al. Effect of intravenous albumin on renal impairment and mortality in patients with cirrhosis and spontaneous bacterial peritonitis. N Engl J Med 1999;341:403-9.

4 Ginès $A$, Fernández-Esparrach $G$, Monescillo $A$, et al. Randomized trial comparing albumin, dextran 70, and polygeline in cirrhotic patients with ascites treated by paracentesis. Gastroenterology 1996;111:1002-10.

5 Angeli $P$, Fasolato $S$, Mazza $E$, et al. Combined versus sequential diuretic treatment of ascites in non-azotaemic patients with cirrhosis: results of an open randomized clinical trial. Gut 2010;59:98-104.

6 Mehta RL, Kellum JA, Shah SV, et al. Acute Kidney Injury Network: report of an initiative to improve outcomes in acute kidney injury. Crit Care 2007;11:R31.

7 Joannidis M, Metnitz B, Bauer P, et al. Acute kidney injury in critically ill patients classified by AKIN versus RIFLE using the SAPS 3 database. Intensive Care Med 2009;35:1692-702.

8 Piano S, Rosi S, Maresio G, et al. Evaluation of the Acute Kidney Injury Network criteria in hospitalized patients with cirrhosis and ascites. J Hepatol 2013;59:482-9.

9 Fagundes C, Barreto R, Guevara M, et al. A modified acute kidney injury classification for diagnosis and risk stratification of impairment of kidney function in cirrhosis. J Hepatol 2013;59:474-81.

10 Belcher JM, Garcia-Tsao G, Sanyal AJ, et al. Association of AKI with mortality and complications in hospitalized patients with cirrhosis. Hepatology 2013;57:753-62.

11 de Carvalho JR, Villela-Nogueira CA, Luiz RR, et al. Acute kidney injury network criteria as a predictor of hospital mortality in cirrhotic patients with ascites. J Clin Gastroenterol 2012;46:e21-26.

12 Kidney Disease: Improving Global Outcomes (KDIGO) Clinical Practice Guideline for Acute Kidney Injury. Kidney Int Supp/ 2012;2:1-138.

13 Fine JP, Gray RJ. A proportional hazards model for the subdistribution of a competing risk. J Am Stat Assoc 1999;94:496-509.

14 Arroyo V, Gines P, Gerbes AL, et al. Definition and diagnostic criteria of refractory ascites and hepatorenal syndrome in cirrhosis. Hepatology 1996;23:164-76.

15 Salerno $F$, Gerbes $A$, Gines $P$, et al. Diagnosis, prevention and treatment of the hepatorenal syndrome in cirrhosis. Gut 2007;56:1310-8.

16 Durand F, Valla D. Assessment of the prognosis of cirrhosis: child-Pugh versus MELD. J Hepatol 2005;42(Suppl 1):S100-107.

17 Wiesner R, Edwards E, Freeman R, et al. United Network for Organ Sharing Liver Disease Severity Score Committee. Model for end-stage liver disease (MELD) and allocation of donor livers. Gastroenterology 2003;124:91-6.

18 D'Amico G, Garcia-Tsao G, Pagliaro L. Natural history and prognostic indicators of survival in cirrhosis: a systematic review of 118 studies. J Hepatol 2006;44:217-31.

19 Broce JC, Price LL, Liangos 0 , et al. Hospital-acquired acute kidney injury: an analysis of nadir-to-peak serum creatinine increments stratified by baseline estimated GFR. Clin J Am Soc Nephrol 2011;6:1556-65.

20 Martín-Llahí M, Guevara M, Torre A, et al. Prognostic importance of the cause of renal failure in patients with cirrhosis. Gastroenterology 2011;140:488-96. 


\section{GUT Acute kidney injury and acute-on-chronic liver failure classifications in prognosis assessment of patients with acute decompensation of cirrhosis}

Paolo Angeli, Ezequiel Rodríguez, Salvatore Piano, Xavier Ariza, Filippo Morando, Elsa Solà, Antonietta Romano, Elisabet García, Marco Pavesi, Alessandro Risso, Alexander Gerbes, Chris Willars, Mauro Bernardi, Vicente Arroyo and Pere Ginès

Gut 2015 64: 1616-1622 originally published online October 13, 2014 doi: 10.1136/gutjnl-2014-307526

Updated information and services can be found at:

http://gut.bmj.com/content/64/10/1616

These include:

Supplementary Supplementary material can be found at:

Material http://gut.bmj.com/content/suppl/2014/10/13/gutjnl-2014-307526.DC1

References This article cites 20 articles, 3 of which you can access for free at: http://gut.bmj.com/content/64/10/1616\#BIBL

Email alerting Receive free email alerts when new articles cite this article. Sign up in the service box at the top right corner of the online article.

ErrataAn erratum has been published regarding this article. Please see next page or: /content/65/8/1394.full.pdf

Notes

To request permissions go to:

http://group.bmj.com/group/rights-licensing/permissions

To order reprints go to:

http://journals.bmj.com/cgi/reprintform

To subscribe to BMJ go to:

http://group.bmj.com/subscribe/ 


\section{Erratum: Acute kidney injury and acute-on- chronic liver failure classifications in prognosis assessment of patients with acute decompensation of cirrhosis}

Angeli P, Rodríguez E, Piano S, et al. Acute kidney injury and acute-on-chronic liver failure classifications in prognosis assessment of patients with acute decompensation of cirrhosis. Gut 2015;64:1616-22. The funding statement has been updated to read: Part of the work reported in this study has been funded by the project PI12/00330, integrated in the Plan Nacional I+D $+\mathrm{I}$ and cofunded by ISCIII-Subdirección General de Evaluación and European Regional Development Fund (ERDF).

Gut 2016;65:1394. doi:10.1136/gutjnl-2014-307526corr1 